\title{
Analysis of chromosomal aberrations in workers exposed to low level benzene
}

\author{
A Yardley-Jones, Diana Anderson, D P Lovell, P C Jenkinson
}

\begin{abstract}
Metaphase chromosome preparations were analysed as part of a larger study from a population occupationally exposed to benzene and compared with a control group. Forty eight of the 66 exposed individuals and 29 of the 33 controls had samples in which metaphase spreads could be evaluated. The incidence of chromosomal aberrations (particularly chromatid deletions and gaps) in the exposed group were slightly increased compared with the control group. This increase was of borderline significance in parametric statistical tests but was significant using Fisher's exact test. No lifestyle factors had any consistent effect on the incidence of chromosome aberrations, although there was a small reduction in gaps with increasing cigarette smoking. Older individuals had a higher incidence of chromosome exchanges and "other" aberrations. Individuals who reported a recent viral illness had a higher incidence of aberrations particularly gaps. There was no evidence of any correlation in the incidence of chromosome aberrations with any of the other biological parameters previously reported. The increased incidence of aberrations seen in the group exposed to benzene may result from a history of exposure to benzene. Nevertheless, other explanations such as sampling, interindividual variability, and unintentional bias in the selection of two groups cannot be excluded.
\end{abstract}

It is now well known that benzene shows genotoxic effects as it is able to induce chromosomal aberrations in animals and man at relatively high levels of exposure. ${ }^{1}$ Sarto et al examined the peripheral blood lymphocytes from 22 healthy workers from benzene, toluene, and xylene production factories. ${ }^{2}$ Exposures

Occupational Health Division, Shell-Mex House, London

A Yardley-Jones

British Industrial Biological Research Association, Carshalton, Surrey SM5 4DS

D Anderson, D P Lovell, P C Jenkinson were assessed from peripheral air samples, expired alveolar air, and urinary phenol concentrations. The values ranged from $0 \cdot 2$ to 12.4 parts per million time weighted average but exposures before 1971 were reported as higher. Subjects were matched for absence of exposure to other genotoxic agents, as well as social variables including medical history, smoking, and eating habits. The chromosome data showed a small increase in the chromosome type aberrations in the benzene workers but not in chromatid type aberrations. The authors believed that this may have resulted from either recent exposure to low concentrations of benzene or to past exposures.

Clare et al analysed chromosomes of peripheral blood lymphocytes from 10 workers after a single episode of high exposure to benzene. ${ }^{3}$ Workers on a single shift were exposed to high concentrations of benzene because of a spillage of about 1200 gallons of benzene during the loading of a ship. Substantial concentrations of phenol were found in the workers' urine after the spillage, indicating a high peak exposure. Chromosomal analyses were carried out on the 10 men exposed three months after the spillage and compared with the 11 men employed on a comparable shift but who were not highly exposed. No significant difference was detected in the incidence of chromosomal aberrations between the two groups.

The study on chromosomal aberration data reported here is part of a larger study examining other genotoxic end points in a population occupationally exposed to benzene. ${ }^{45}$ The exposure was believed to be relatively low (less than $10 \mathrm{ppm}$ ) although occasionally workers experienced short term peak exposures up to about $100 \mathrm{ppm}$ level. ${ }^{45}$

\section{Materials and methods}

SAMPLE POPULATION

Peripheral blood lymphocytes from 66 men with low average exposure to benzene and 33 control men were investigated for the frequency of chromosomal aberrations.

The 66 men worked in jobs where benzene or material containing benzene was handled, and all had been potentially exposed to benzene for over five years. Three main work areas were identified: shipping, oil movements, and aromatic production. Exposures in this study were assumed to be similar to 
those of previous personal and workplace air samples collected over a period of years from individuals in similar jobs. The exposure data were classified according to shift time weighted average (TWA) and level. Values for the TWA of benzene were 1-10 ppm and for the level of benzene 10-100 ppm. Details of the classification and various categories are reported elsewhere. ${ }^{45}$ Some jobs such as loading and unloading of benzene on the jetty had strict requirements for the wearing of protective breathing apparatus. In other jobs the wearing of respiratory protection was sporadic. Control individuals were selected because of their presumed non-exposure to known genotoxic agents. Each individual completed a detailed questionnaire on health status, social habits such as smoking and drinking, exposure to diagnostic ionising radiation, and recent viral infection.

\section{BLOOD SAMPLE COLLECTION AND CULTURE}

Samples of $5 \mathrm{ml}$ heparinised blood were collected from eight individuals a week by venepuncture during the early morning and stored at room temperature for up to six hours before starting blood cultures. For each sample, $0.8 \mathrm{ml}$ of freshly remixed blood was added to each of two plastic universal bottles containing $10 \mathrm{ml}$ of Eagle's minimal essential medium (Gibco). Cells were sedimented by centrifugation $(170 \times \mathrm{g}, 5 \mathrm{~min})$, the supernatant withdrawn and discarded. The cell pellet (about $0.5 \mathrm{ml}$ ) was disrupted and resuspended in $9.5 \mathrm{ml}$ medium supplemented with $15 \%$ fetal calf serum (Sera-Lab, Crawley, Sussex) and $50 \mathrm{IU} / \mathrm{ml}$ penicillin $/ 50 \mu \mathrm{g} / \mathrm{ml}$ streptomycin (Gibco). Then $100 \mu$ l of reconstituted phytohaemagglutinin (PHA-R, Wellcome) was added per culture. The cultures were incubated at $37^{\circ} \mathrm{C}$ in humidified $5.0 \pm 0.1 \% \mathrm{CO}^{2}$ in air.

\section{CELL HARVEST AND SLIDE PREPARATION}

After 48 hours incubation, $50 \mu \mathrm{l}$ of $10 \mu \mathrm{g} / \mathrm{ml}$ demecolcine (Sigma) was added to each culture. After two hours reincubation cells were harvested by centrifugation, swelled in $0.075 \mathrm{M} \mathrm{KCl}$, and fixed in repeated changes of 3:1 methanol and glacial acetic acid. Chromosome spreads were prepared using the air drying technique and stained with $5 \%$ Giemsa (Gurr's, BDH) for five minutes. Preparations were mounted in DePex (Gurr's, BDH). Where possible 50 metaphase spreads per culture were scored for chromosome aberrations (100 per individual). Aberrations were classified according to a simplified version of that recommended by the United Kingdom Environmental Mutagen Society. ${ }^{6}$

\section{STATISTICAL ANALYSIS}

The data were analysed by a series of statistical methods. Comparison between replicate cultures within an individual were carried out by Fisher's exact tests. Comparisons between groups exposed to differing "lifestyle" factors such as smoking or drinking were made using analysis of variance or pooled two sample $t$ tests after arcsin transformation of the proportion of the individual's cells that contained the class of aberration. Comparisons between the groups of exposed and control individuals were made using analysis of variance methodology and by Fisher's exact tests. In the latter case tests of the homogeneity of the proportions of aberrations within the control and exposed groups were carried out using the chi-squared test of homogeneity of binomial values. Multiple linear regression was also used to investigate all the lifestyle factors simultaneously.

The data for each individual were summarised by expressing the number of aberrations as the total aberrations observed or as the number of cells showing aberrations. In both cases the statistics were calculated either including or excluding gaps.

\section{Results}

Of the 100 individuals recruited for the study, 99 completed a questionnaire. Cultures were obtained from 22 from which no metaphases could be scored. This left samples from 48 exposed and 29 controls where spreads could be evaluated. Sixty individuals had two cultures that could be-scored and 17 only one culture.

\section{VARIABILITY BETWEEN DUPLICATE CULTURES}

There was no evidence of significant differences between the duplicate cultures for any of the classes of aberrations from the 60 individuals using two sided Fisher's exact tests.

\section{EFFECT OF LIFESTYLE FACTORS ON CHROMOSOME ABERRATION FREQUENCIES}

Each of the classes of aberration was analysed using analysis of variance on the arcsin transformed proportion of aberrations per cell. One way analyses of variance were carried out based on the classification of the responses to the questionnaire. The considerable number of analyses performed, together with the potential confounding effects of the various different factors, made this statistical approach unsuitable for precise significance testing but useful for exploratory data analysis.

There were no significant analyses of variance of differences in the number of gaps apart from the analysis using presence or absence of recent viral illness as a factor. The five subjects responding to this question had slightly raised numbers of gaps $\left(F_{(1,75)}=12.66 \mathrm{p}<0.001\right.$; transformed mean and SD $8.86 \pm 3.15 n=5 v 2.93 \pm 3.63 n=72$ ). Most of this effect, however, was probably traceable to an individual where three gaps were found in the 50 cells from the single culture available for scoring.

Significant variation between batches in the total number of aberrations including gaps was detected $\left(F_{(1,65)}=2.79 \mathrm{p}<0.01\right)$. This resulted from the three individuals from two batches (Nos 5 and 6 ) where 
Table 1 Total number of aberrations in control and exposed individuals

\begin{tabular}{lccc}
\hline & $\begin{array}{c}\text { Control } \\
(\boldsymbol{n}=29)\end{array}$ & $\begin{array}{c}\text { Exposed } \\
(\boldsymbol{n}=48)\end{array}$ & $\begin{array}{c}\text { Total } \\
(\boldsymbol{n}=77)\end{array}$ \\
\hline Cells scored & 2481 & 4255 & 6736 \\
Gaps & 13 & 36 & 49 \\
Chromosome deletions & 14 & 32 & 46 \\
Chromosome exchanges & 1 & 5 & 6 \\
Chromatid deletions & 11 & 33 & 44 \\
Chromatid exchanges & 0 & 0 & 0 \\
Hyperdiploidy & 0 & 5 & 5 \\
Others & 2 & 0 & 2 \\
Total aberrations (+ gaps) & 41 & 111 & 152 \\
Total aberrations (- gaps) & 28 & 75 & 103 \\
Cells with aberrations (+ gaps) & 37 & 103 & 140 \\
Cells with aberrations (- gaps) & 25 & 69 & 94 \\
\hline
\end{tabular}

cells could be scored having no aberrations (or gaps) and from higher frequencies in individuals in two other batches. Reanalysis excluding the three individuals from batches 5 and 6 reduced but did not remove the statistical significance. The difference between individuals reporting a viral illness and the rest was again significant $\left(F_{(1,75)}=4.39\right)$ probably because of the number of gaps in the culture from the single individual mentioned previously.

\section{COMPARISON OF INDIVIDUALS EXPOSED TO BENZENE WITH CONTROL GROUP}

Table 1 shows the total number of chromosomal aberrations in the control and exposed individuals. The two groups were compared in two ways: firstly, by pooled two sample $t$ tests on the arcsin transformed proportion and, secondly, by Fisher's exact tests of the actual numbers of classes of aberrations. Both tests were one sided, testing the hypothesis of increased incidence of aberrations in the exposed individuals. The parametric $t$ test approach showed no significant differences between the two groups although in some cases such as the proportion of cells showing aberrations including gaps the $p$ value (table 2) was close to significance in a one sided test $(p=0.05)$. In all classes of aberrations except those classified as others the proportion of cells with aberrations were higher in the exposed than in the control group.

Analysis of the actual number of cells showing aberrations using Fisher's exact test showed highly significant increases in the exposed group when all classes of aberrations were included especially when
Table 3 Chi-squared $\left(\chi^{2}\right)$ test of homogeneity within control and exposed groups

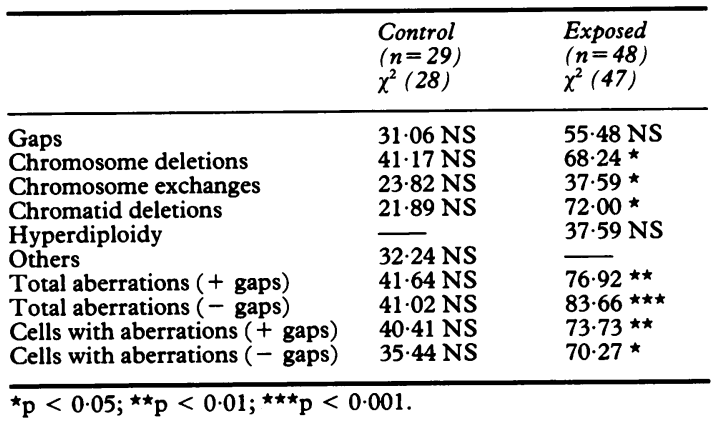

gaps were included in the analysis $(p=0.023$ and $\mathrm{p}=0.006$ respectively). Several of the individual aberration classes were close to significance at the $p=0.05$ level, particularly chromatid deletions $(p=0.07)$. Such an analysis assumes that the individuals within the group are homogeneous in their responses. Tests for homogeneity (chi-squared) tests were carried out and significant heterogeneity detected within the exposed group (table 3 ).

The transformed proportion of cells showing various classes of aberration were correlated with various other biological parameters measured in the same study - that is, maximum mitogenic response to phytohaemagglutinin, mean corpuscular volume, urinary phenol concentration, sister chromatid exchange (SCE) frequencies, and a cell cycle kinetic index (proliferative rate index) from the SCE preparations. No significant correlations were detected except between the different classes of aberrations and the aggregate measures of aberrations. The correlation, for instance, between the transformed proportion of cells with aberrations including gaps and the SCE frequency of the same individuals was 0.15 (only 46 individuals had measures for both SCE frequencies and chromosome aberrations).

\section{Discussion}

Individuals exposed to a low level of benzene showed a slightly increased incidence of chromosomal aberrations (mainly chromatid deletions and gaps). The average incidence of cells with aberrations including gaps was $2.5 \%$ for the exposed and $1.67 \%$

Table 2 Average aberrations per 100 cells from control and exposed individuals

\begin{tabular}{|c|c|c|c|c|c|c|c|c|c|c|c|c|c|}
\hline & \multirow[b]{2}{*}{ No } & \multirow{2}{*}{$\begin{array}{l}\text { Cells } \\
\text { scored }\end{array}$} & \multirow[b]{2}{*}{ Gaps } & \multicolumn{2}{|c|}{ Chromosomes } & \multicolumn{2}{|c|}{ Chromatids } & \multirow{2}{*}{$\begin{array}{l}\text { Hyper- } \\
\text { diploid }\end{array}$} & \multirow[b]{2}{*}{ Others } & \multicolumn{2}{|c|}{ Total aberrations } & \multicolumn{2}{|c|}{ Cells with aberrations } \\
\hline & & & & Deletions & Exchanges & Deletions & Exchanges & & & $(+g a p s)$ & $(-g a p s)$ & $(+$ gaps $)$ & $(-$ gaps $)$ \\
\hline $\begin{array}{l}\text { Control } \\
\text { SD } \\
\text { Exposed } \\
\quad \text { SD } \\
\text { Pooled } t \text { test } \\
\text { Fisher's exact } \\
\text { test }\end{array}$ & $\begin{array}{l}29 \\
48\end{array}$ & $\begin{array}{r}85.6 \\
\pm 22.1 \\
88.6 \\
\pm 21.8\end{array}$ & $\begin{array}{r}0.59 \\
\pm 0.87 \\
0.90 \\
\pm 1.21 \\
0.09 \\
0.09\end{array}$ & $\begin{array}{r}0.65 \\
\pm 1.14 \\
0.73 \\
\pm 1.09 \\
0.26 \\
0.23\end{array}$ & $\begin{array}{r}0.03 \\
\pm 0.19 \\
0.10 \\
\pm 0.31 \\
0.14 \\
0.29\end{array}$ & $\begin{array}{r}0.50 \\
\pm 0.71 \\
0.81 \\
\pm 1.28 \\
0.21 \\
0.07\end{array}$ & $\begin{array}{l}0.00 \\
\frac{0}{0.00} \\
- \\
-\end{array}$ & $\begin{array}{c}0.00 \\
\overline{0.10} \\
\pm 0.31 \\
\overline{0.10}\end{array}$ & $\begin{array}{c}0.10 \\
\pm 0.41 \\
0.00 \\
- \\
1.00\end{array}$ & $\begin{array}{l}1.88 \\
\pm 2.21 \\
2.65 \\
\pm 2.45 \\
0.07 \\
0.006^{\star \star}\end{array}$ & $\begin{array}{r}1.29 \\
\pm 1.87 \\
1.75 \\
\pm 1.90 \\
0.09 \\
0.02 \star\end{array}$ & $\begin{array}{l}1.67 \\
\pm 1.85 \\
2.50 \\
\pm 2.36 \\
0.05 \\
0.006 \star \star\end{array}$ & $\begin{array}{r}1.12 \\
\pm 1.45 \\
1.63 \\
\pm 1.79 \\
0.08 \\
0.02 \star\end{array}$ \\
\hline
\end{tabular}


for the control individuals. The corresponding percentages with gaps excluded were 1.63 and $1.12 \%$.

Much of the difference between the two groups could be traced to a few exposed individuals who had a higher incidence of aberrations. The presence of such heterogeneity, especially in the exposed group, may cause an overestimate of the significance level associated with the chi-squared or Fisher's exact tests. This has been recognised by Weil ${ }^{7}$ and others for some time and results from a more similar chance of aberrations in cells from the same individual than in cells from two different individuals. If the exposed individual with the highest frequency of aberrations (three chromatid deletions, one chromosome deletion, and two gaps in 50 cells scored) is excluded from the analysis then the significance of the comparison is reduced appreciably. In the case of cells with aberrations including gaps the probability associated with Fisher's exact test changes from 0.006 to 0.012 and excluding gaps from 0.023 to 0.039 .

The alternative analyses such as the parametric $t$ tests or non-parametric Mann-Whitney $U$ test gave borderline significance at the $p=0.05$ level. Such tests may estimate significance inaccurately when there are a high proportion of zeros or small values in the data.

The increased incidence of aberrations in the exposed individuals therefore results from the heterogeneity of the exposed group. Whether this is a consequence of sampling variability or represents a subgroup of either more exposed or more sensitive individuals cannot be determined. Further analysis of the work history records of those individuals who had the highest number of aberrations provided no extra information to explain the differences. The sample sizes available in this study do not allow statistical tests with sufficient power to detect a $50 \%$ increase over the control incidence of chromosome aberration with any confidence.

Other factors that might account for the variability between individuals were investigated but there was little evidence that lifestyle factors influenced the incidence of aberrations, apart from a few isolated observations. Individuals reporting recent viral illnesses had higher incidences of aberrations; a high proportion of chromosome exchanges were found in the oldest individuals in the study and there were significant reductions in two batches where cultures could be scored from only three of the 16 individuals sampled.

Step wise multiple linear regression of chromosomal aberration data using lifestyle factors identified exposure to benzene and a recent viral infection as a possible explanation for the incidence of aberrations. The best fitting regression line, however, explained only a small proportion of the variation in 77 individuals (approximately 10\%). A similar analysis also showed a significant reduction in gaps with an increased history of smoking. The biological significance, if any, of this finding is unknown.
The results from other biological parameters measured in this study showed no differences between the exposed and control groups. ${ }^{45}$ Some of these parameters are thought to reflect relatively recent genotoxic insults. It can be argued, therefore, that exposures at, and for a short time before, the study were not responsible for the differences seen between the groups in the chromosomal aberration data. Owing to the long half life of lymphocyte populations, the accumulation of genetic damage by lymphocytes allows monitoring of chronic as well as acute effects from exposure to clastogenic agents. The increased incidence of aberrations in the exposed group, if related to exposure to benzene, may have resulted from higher exposures in the past. These exposures, though, were within the relatively higher exposure limits then permitted.

Follow up studies to investigate possible benzene related effects are difficult to carry out in these types of investigation. Possibly more detailed analysis of the material using chromosomal banding techniques could have shown more subtle effects resulting from the more recent lower limit exposure. Such an analysis was, unfortunately, not practicable with this study.

We thank all the employees who took part in the study; Professor D H Elliott, Dr N I P McKie, and Dr A C MacLean for their encouragement and support; Mr I Smith for preparing the blood samples; Dr R C Dewdney, Mrs S K Doubell, and Mrs L Smith for help in processing and analysing the samples; and Dr M K Molyneux for supplying exposure data and preparing exposure classifications. We are also grateful to Mrs J Groves for typing and preparing the manuscript.

Requests for reprints to: Dr A Yardley-Jones, BIBRA, Carshalton, Surrey SN5 4DS.

1 Dean BJ. Recent findings on the genetic toxicology of benzene, toluene, xylenes and phenols. Mutat Res 1985;154:153-81.

2 Sarto F, Cominato I, Pinton AM, et al. A cytogenetic study on workers exposed to low concentrations of benzene. Carcinogenesis 1984;5:827-32.

3 Clare MG, Yardley-Jones A, MacLean AC, Dean BJ. Chromosome analysis from peripheral blood lymphocytes of workers after an acute exposure to benzene. Br J Ind Med 1984;41:249-53.

4 Yardley-Jones A, Anderson D, Jenkinson PC. Effect of occupational exposure to benzene on phytohaemaglutinin (PHA) stimulated lymphocytes in man. Br J Ind Med 1988;45:516-22.

5 Yardley-Jones A, Anderson D, Jenkinson PC, Lovell DP, Blowers SD, Davis MJ. Genotoxic effects in peripheral blood and urine of workers exposed to low level benzene. $B r J$ Ind Med 1988;45:694-700.

6 Scott D, Danford N, Dean B, Kirkland D, Richardson C. In vitro chromosome aberrations assays. In: Dean BJ, ed. UKEMS sub-committee guidelines for mutagenicity testing. Part I. Basic test battery. Swansea: United Kingdom Environmental Mutagen Society, 1983:41-64.

7 Weil CS. Selection of the valid number of sampling units and a consideration of their combination in toxicological studies involving reproduction, teratogenesis or carcinogenesis. Food and Cosmetic Toxicology 1970;8:177-82.

Accepted 13 March 1989 